\title{
Modeling Basket Credit Default Swaps with Default Contagion
}

\author{
Helen Haworth* \\ Christoph Reisinger \\ haworthh@maths.ox.ac.uk_reisinge@maths.ox.ac.uk \\ The Nomura Centre for Quantitative Finance \\ OCIAM Mathematical Institute \\ Oxford University \\ 24-29 St Giles \\ Oxford, OX1 3LB \\ England \\ Tel. +44 1865280612
}

December 2006

${ }^{*}$ This work is kindly supported by Nomura and the EPSRC. 


\section{Executive Summary}

The specification of a realistic dependence structure is key to the pricing of multi-name credit derivatives. We value small $k^{t h}$-to-default CDS baskets in the presence of asset correlation and default contagion. Using a first-passage framework, firm values are modeled as correlated geometric Brownian motions with exponential default thresholds. Idiosyncratic links between companies are incorporated through a contagion mechanism whereby a default event leads to jumps in volatility at related entities. Our framework allows for default causality and is extremely flexible, enabling us to evaluate the spread impact of firm value correlations and credit contagion for symmetric and asymmetric baskets.

\section{Keywords}

Basket credit default swaps, default dependence, credit risk, correlation, contagion, firstpassage model. 


\section{Introduction}

The effects of a corporate default are usually felt far beyond the individual firm in question, rippling through the market, causing changes in the credit spreads of related companies. Generally these would be increases in spreads of companies deemed to be exposed to similar risks, but could also be manifest as tightening spreads of companies likely to benefit from the default. In this paper, we specify a multi-firm structural credit model with a dependence structure incorporating firm-value correlations and this type of contagious default behavior.

Broadly speaking, credit weakness, ratings downgrades and ultimately corporate default can occur in three main ways. Firstly, a company may be adversely affected for reasons specific to that company alone (e.g. poor financial management). Secondly, credit weakness may occur due to a factor or factors impacting multiple companies - whether in the form of a cyclical influence related to the economy, or a market-wide shock such as an earthquake or terrorist attack. Finally, companies are related through ties, some of which are real (e.g. a trade-creditor agreement), others of which are purely a matter of perception - for example the fear of accounting fraud. Credit dependence then occurs primarily through two mechanisms - either as a direct consequence of a common driving factor, or due to inter-company ties.

In order to incorporate such a dependence structure into the structural framework, we consider a firm-value model with default as the first hitting time of an exponential barrier. Firm assets are modeled as correlated geometric Brownian motions and default by one firm has a knockon impact on remaining companies in the form of a jump in firm volatility - contagion. In this way, we are able to capture the two facets of the dependence structure. The correlation in firm values reflects a longer-term common driving influence on corporate strength whilst default contagion represents a direct link between the fortunes of both companies. The result is a model that allows for default causality and is asymmetric with regard to default risk, a significant improvement on prior models.

Until recently, the vast majority of work on the structural model focused on the case of a single firm, with two popular commercial packages, Moody's KMV and CreditGrades ${ }^{T M}$ motivated by the single-firm structural model ${ }^{1}$. Giesecke (2004), Schönbucher (2003) and Lando (2004) provide a good introduction to structural models, their development since first introduced by Merton (1974), and their traditional place within credit modeling. Though not so straightforward mathematically, structural models are far more grounded in economic fundamentals than many other models and thus form a good starting point for a realistic description of credit dynamics. Very little, however, has been published for multiple companies, with the market mainly focused on copulas or conditionally independent factor models ${ }^{2}$ in the multivariate case. In two exceptions, Hull and White (2000) use Monte Carlo simulation to value a basket CDS in a structural-type model whilst Zhou (2001) calculates default correlations for two correlated Brownian motions.

Existing models used in the market suffer many limitations and as problems have arisen with their widespread use, structural models have started to see some renewed interest. Hull et al.

\footnotetext{
${ }^{1}$ Further details of these approaches can be found at www.moodyskmv.com and in Finger et al. (2002), respectively.

${ }^{2}$ For a good overview of the use of copulas in finance, see Cherubini et al. (2004); Schönbucher (2003) provides a useful summary and references for factor models.
} 
(2005) price CDO tranches in a structural framework where assets are driven by a common factor. In another approach, Luciano and Schoutens (2005), Moosbrucker (2006) and Baxter (2006) assume that firm values are driven by Levy processes rather than geometric Brownian motions and model credit derivatives using Variance Gamma processes. Haworth et al. (2006) build on the approach of Zhou (2001) to derive closed-form solutions for corporate bond yields in the presence of default contagion and correlated two-firm basket credit default swaps.

This paper is a generalisation of the analytical approach of Haworth et al. (2006), incorporating a more flexible and realistic specification of the default contagion mechanism and allowing for rapid valuation of three-firm credit default swap (CDS) baskets. It is organised as follows. Section 2 contains a description of the model and its numerical implementation, with results for two-firm baskets considered in detail in Section 3. The two-company model is extended in Section 4 to allow the impact of default contagion to dissipate over time. Results for three-firm baskets are then illustrated in Section 5 and the current numerical limitations of the model in higher dimensions are discussed. Section 6 outlines the extension to a general $m$-factor framework and we conclude with a discussion of future areas of interest.

\section{Modeling Methodology}

\subsection{Setup}

We consider $n$ companies with firm values $V_{i}, i=1 \ldots n$. For each company, firm value is assumed to follow a geometric Brownian motion, with default as the first time that the value of the firm hits a lower default barrier $b_{i}(t)$. As in Merton (1974) and Black and Cox (1976), we assume that a firm's value can be constructed from tradable securities and so under the risk-neutral pricing measure, for $i=1, \ldots, n$,

$$
\mathrm{d} V_{i}(t)=\left(r_{f}-q_{i}\right) V_{i}(t) \mathrm{d} t+\sigma_{i} V_{i}(t) \mathrm{d} W_{i}(t)
$$

where the risk-free rate, $r_{f}$, dividend yields, $q_{i}$, and volatilities, $\sigma_{i}$, are constants, $W_{i}(t)$ are Brownian motions and $\operatorname{cov}\left(W_{i}(t), W_{j}(t)\right)=\rho_{i j} t$ for constant correlations $\rho_{i j}, i, j=1, \ldots, n$.

We assume that each company has an exponential default barrier, reflecting the existence of debt covenants, and denote the default barrier for company $i$ by

$$
b_{i}(t)=K_{i} e^{-\gamma_{i}(T-t)}
$$

for constants $K_{i}, \gamma_{i}$. T represents the maturity of the product of interest or, more generally, the length of the time period under consideration.

We are interested in default probabilities - for example, the probability that a given firm defaults by a given time horizon, or the probability that a certain number of firms default in a specified period. If $\Omega$ represents the event of interest, $\mathbf{V}$ is the vector of firm values and $\mathcal{L}$ is the infinitesimal generator of (1) then we can calculate the probability of the event $\Omega$ by solving

$$
\begin{array}{r}
\mathcal{L} U=\frac{\partial U}{\partial t}+\sum_{i=1}^{n} \beta_{i} V_{i} \frac{\partial U}{\partial V_{i}}+\frac{1}{2} \sum_{i, j=1}^{n} a_{i j} V_{i} V_{j} \frac{\partial^{2} U}{\partial V_{i} \partial V_{j}}=0 \\
U(\mathbf{V}, T)=\mathbb{I}_{\Omega}(\mathbf{V}(T))
\end{array}
$$


for the function $U(\mathbf{V}, t)$, since by the Feynman-Kac formula,

$$
U(\mathbf{v}, t)=\mathbb{E}\left\{\mathbb{I}_{\Omega}(\mathbf{V}(T)) \mid \mathbf{V}(t)=\mathbf{v}\right\}=\mathbb{P}(\mathbf{V}(T) \in \Omega \mid \mathbf{V}(t)=\mathbf{v}),
$$

where $\mathbb{I}_{\Omega}$ denotes the indicator function of the event $\Omega$ and

$$
\begin{aligned}
\beta_{i} & =r_{f}-q_{i} \\
a_{i j} & =\rho_{i j} \sigma_{i} \sigma_{j} .
\end{aligned}
$$

\subsection{Introducing Credit Contagion}

As specified so far, the dependence structure is driven purely by the correlation in firm values. Default by a company has no direct impact on the behavior of the remaining companies; there is no credit or default contagion. This we incorporate by allowing the values of $a_{i j}$ in (2), to jump on default. If company $i$ defaults, this causes a jump in the volatilities, $\sigma_{j}$, of firms $j$, $i \neq j$, thereby introducing credit contagion. Subsequent defaults cause further jumps in the volatilities of firms that are still 'alive'.

By relating the size and direction of the jump in $\sigma_{j}$ to the correlation between companies $i$ and $j$, both positive and negative effects and differing degrees of contagion can be incorporated. The result is a dependence structure incorporating both mechanisms described in the introduction. Exposure to common factors arises through the specification of correlated firm value processes in (1), whilst the contagion mechanism reflects the existence of a network of inter-company links.

Asymmetric ties are easy to incorporate, allowing some companies to have greater influence than others, in the vein of work done by Jarrow and Yu (2001) in which 'primary' companies impact 'secondary' companies but not vice versa ${ }^{3}$. Our model is flexible enough to specify an arbitrary number of different relationships between companies.

\subsection{Implementation}

We solve (2) backwards in time on $[0, T] \times \mathbb{R}_{+}^{n}$ using a finite-difference method with Crank Nicolson time-stepping and a multigrid solver as in Reisinger and Wittum (2004). To define the initial condition, we specify the coefficients in equation (2) such that once a company hits its default barrier it remains constant. In other words, we set each firm's drift and volatility to zero on its barrier and define new coefficients $\widetilde{\beta}_{i}$ and $\widetilde{a}_{i j}$,

$$
\begin{aligned}
& \widetilde{\beta}_{i}\left(V_{i}, t\right):=\beta_{i} \mathbb{I}_{\left\{V_{i}(t)>b_{i}(t)\right\}} \\
\widetilde{a}_{i j}\left(V_{i}, V_{j}, t\right):= & a_{i j} \mathbb{I}_{\left\{V_{i}(t)>b_{i}(t), V_{j}(t)>b_{j}(t)\right\}} .
\end{aligned}
$$

In this way, when a firm reaches its default barrier and defaults, its value does not change, enabling us to count the number of companies whose values are less than or equal to their default barriers ${ }^{4}$ at a given point in time and define the initial condition in (3) according to

\footnotetext{
${ }^{3} \mathrm{~A}$ 'primary' company is likely to be larger with a greater market impact than a 'secondary' company. For example Microsoft or General Motors compared to a small, local IT or auto component manufacturer.

${ }^{4}$ The barrier is an increasing function, so its value at maturity will be greater than or equal the value at which default occurred.
} 
the value we want to calculate. For example, if we want the probability that all companies survive, we set the initial condition to be one when there are no companies at or below their default barriers, and zero elsewhere. If we want the probability of $k$ defaults, we set it to be one when $k$ companies are valued at or below their barriers, zero otherwise.

The event that the $k \leq n$ companies $i_{1}, \ldots, i_{k}$ default by time $T$ (but no others) is given by the set

$$
\Omega_{\left\{i_{1}, \ldots, i_{k}\right\}}=\left\{V_{i_{j}}(T) \leq b_{i_{j}}(T) \quad \text { for } \quad j=1, \ldots, k \quad \text { and } \quad V_{i_{j}}(T)>b_{i_{j}}(T) \text { otherwise }\right\}
$$

and the set $\Omega_{k}$ corresponding to exactly $k$ companies defaulting in $[0, T]$ is

$$
\Omega_{k}=\bigcup_{I \subset\{1, \ldots, n\},|I|=k} \Omega_{I}
$$

We can therefore calculate the probability of $k$ defaults in $[0, T]$ by solving $(2)$ with $U(\mathbf{V}, T)=$ $\mathbb{I}_{\Omega_{k}}(\mathbf{V}(T))$.

To illustrate our approach, we incorporate default contagion by assuming that if company $i$ defaults, the volatility of company $j, i \neq j$, jumps by

$$
\sigma_{j} \rightarrow \sigma_{j} F^{\rho_{i j}}
$$

for some constant $F \geq 1$. In other words,

$$
\sigma_{j}(\mathbf{V}, t)=\left\{\begin{array}{rll}
\sigma_{j} & \text { if } & V_{i}(t)>b_{i}(t), V_{j}(t)>b_{j}(t) \\
\sigma_{j} F^{\rho_{i j}} & \text { if } & V_{i}(t) \leq b_{i}(t), V_{j}(t)>b_{j}(t) \\
0 & \text { if } & V_{j}(t) \leq b_{j}(t)
\end{array}\right.
$$

In this way, a company positively correlated with the defaulting company experiences an increase in volatility, and hence an increase in credit spreads, whilst a negatively correlated company becomes less volatile and consequently less risky. The degree of correlation has an impact on the size of the jump in volatility, and by changing the value of $F$, default can be assumed to have a bigger or smaller influence on the strength of the remaining company. This formulation is intuitive and serves to illustrate the impact that company relationships can have on credit spreads. However, (7) is of course just one of many possible specifications of the contagion mechanism that could be used and others could be handled similarly. A subsequent default by a firm $k \notin\{i, j\}$ would be incorporated in exactly the same way with

$$
\sigma_{j} \rightarrow \sigma_{j} F^{\rho_{i j}} \rightarrow \sigma_{j} F^{\rho_{i j}} F^{\rho_{k j}},
$$

and so on for any further defaults.

In the two dimensional case, we can also solve the problem analytically on the boundary since in the event of default by one company, the equation reduces to a one-dimensional hitting probability. Rather than solving the whole problem numerically as outlined above, it is therefore possible to define the boundary conditions analytically as the solution of the remaining one-firm problem. This approach is outlined in more detail in Section 4, where it is applied to the case of contagion that decays over time. Extension of this method to three or more dimensions could be done recursively, but would be considerably more involved. 


\subsection{CDS Spread Calculation}

The buyer of a $k^{\text {th }}$-to-default credit default swap (CDS) on a basket of $n$ companies pays a premium, the CDS spread, for the life of the CDS - until maturity or the $k^{\text {th }}$ default, whichever happens first. In the event of default by the $k^{t h}$ underlying reference company, the buyer receives a default payment and the contract terminates. Writing $\tau_{k}$ for the time of the $k^{\text {th }}$ default, if recovery on default is $R$, and the protection buyer makes continuous spread payments, $c$, on a par value $K$, then the discounted spread payment (DSP) and discounted default payment (DDP) on the $k^{\text {th }}$-to-default basket are

$$
\begin{aligned}
\mathrm{DSP} & =c K \int_{0}^{T} e^{-r_{f} s} \mathbb{P}\left(\tau_{k}>s\right) \mathrm{d} s \\
\mathrm{DDP} & =(1-R) K \int_{0}^{T} e^{-r_{f} s} \mathbb{P}\left(s \leq \tau_{k} \leq s+d s\right) \mathrm{d} s \\
& =(1-R) K \int_{0}^{T}-e^{-r_{f} s} \frac{\partial}{\partial s} \mathbb{P}\left(\tau_{k}>s\right) \mathrm{d} s \\
& =(1-R) K\left\{1-e^{-r_{f} T} \mathbb{P}\left(\tau_{k}>T\right)-r_{f} \int_{0}^{T} e^{-r_{f} s} \mathbb{P}\left(\tau_{k}>s\right) \mathrm{d} s\right\},
\end{aligned}
$$

giving market $k^{t h}$-to-default CDS spread, $c_{k}$,

$$
c_{k}=\frac{(1-R)\left\{1-e^{-r_{f} T} \mathbb{P}\left(\tau_{k}>T\right)-\int_{0}^{T} r_{f} e^{-r_{f} s} \mathbb{P}\left(\tau_{k}>s\right) \mathrm{d} s\right\}}{\int_{0}^{T} e^{-r_{f}} s \mathbb{P}\left(\tau_{k}>s\right) \mathrm{d} s} .
$$

In addition to calculating default and survival probabilities using (2), we can simultaneously evaluate the integral over time of a function of the default or survival probability of interest. By setting the initial condition to be one if less than $k$ companies default, we can therefore calculate $\mathbb{P}\left(\tau_{k}>T\right)$ and $\int_{0}^{T} e^{-r_{f} s} \mathbb{P}\left(\tau_{k}>s\right) \mathrm{d} s$, and hence $k^{t h}$-to-default credit default swap spreads by $(9)$.

\section{Two-Firm Results}

We begin by illustrating the use and flexibility of the model for the case of two companies, allowing us to highlight its main attributes. By modifying the initial condition, we are able to calculate a number of different survival probabilities and first and second-to-default CDS spreads.

Our results are generated using a regular grid with $2^{10}+1$ grid-points in each direction and 200 time-steps. On an Intel Xeon CPU 3.5 Ghz x2 machine, it takes about eight minutes to generate default probabilities for a given value of correlation at 200 points in a ten-year period. Comparing default probabilities with those obtained analytically in Haworth et al. (2006), using 10 uniform refinements and 200 time-steps gives results accurate to five decimal places. We have gone for accuracy over speed; to generate the same results accurate to three decimal places takes just thirty seconds. 
As a measure of company strength we consider initial credit quality, defined as initial firm value, $V_{i}(0)$, divided by the initial level of the barrier, $b_{i}(0)$. As would be expected, as initial credit quality declines, spreads widen significantly. An indicative scaled distance to default, $\frac{1}{\sigma_{i}} \log \left(V_{i}(0) / b_{i}(0)\right)$, for parameter values of $\sigma_{i}=0.2$ and initial credit quality of 2 is then 3.5. An upward jump in a company's volatility due to default by a related firm therefore reduces the distance to default, leading to higher default probabilities and hence greater spreads.

Figure 1 shows the joint survival probability for five different levels of correlation parameter ${ }^{5}$, $\rho$, and Figure 2 shows the resultant first-to-default CDS spreads. It is worth noting that the presence of contagion has no impact on first-to-default spreads or joint survival probabilities. These values are driven solely by the probability of one of the companies defaulting; what happens later is irrelevant and by definition, default contagion comes into play following the default of the first company. As expected, survival probability declines with time. We also see that survival probability increases with correlation. This is not quite as obvious, but is also the result one would expect. The probability of at least one of the companies defaulting in a given time period is higher when they are negatively correlated than when they are positively correlated. Figure 1 also captures one of the main weaknesses of the structural model, namely that default probabilities over very small time periods are basically zero, and survival probabilities tend to one as time tends to zero, leading to the zero short spreads seen in Figure 2.

Figure 1: Joint survival probability

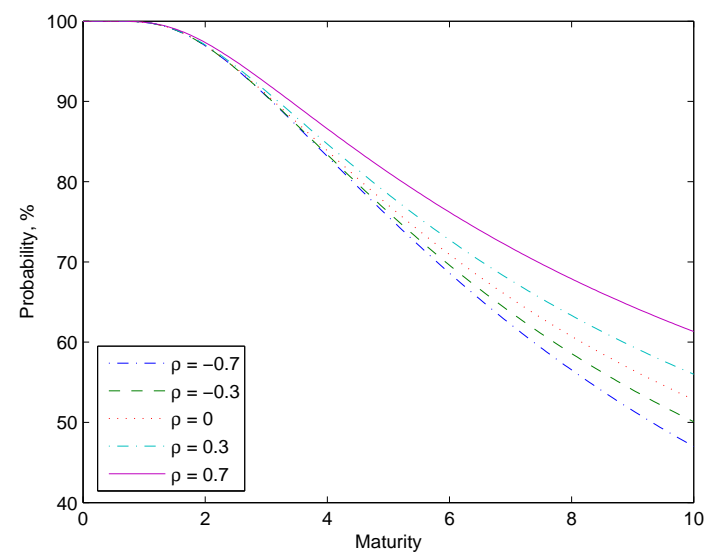

Figure 2: First-to-default CDS spread

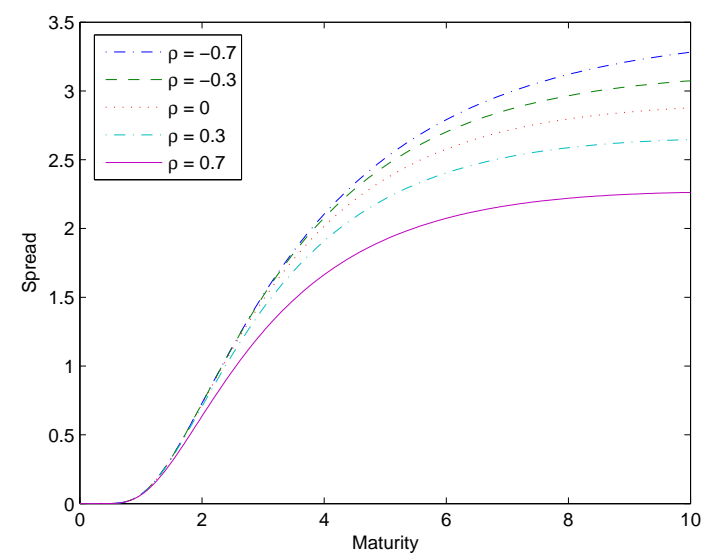

$\sigma_{i}=0.2, r_{f}=0.05, q_{i}=0, \gamma_{i}=0.03$, initial credit quality $=2, \mathrm{R}=0.5$

In Figures 3-8 we consider the impact contagion has on the probability of either one or two defaults occurring. Figures 3 and 4 show the probabilities of exactly one and two defaults respectively, plotted against time for correlation $\rho=+0.5$. Figures $5-8$ give default probabilities against correlation for five-year and ten-year time periods. In each case, $\mathrm{F}=1$ corresponds to no default contagion, whilst increasing $\mathrm{F}$ corresponds to an increasing degree of contagion, with volatility jumping on default according to (7).

Contagion when the companies are negatively correlated has little impact on the probability of one default since the likelihood of a second default is slim. A second default is, however, slightly more likely when there is less contagion, and so the probability of exactly one default

\footnotetext{
${ }^{5}$ Since we have only one correlation parameter in this section, we denote $\rho_{12}=\rho$.
} 
Figure 3: Probability of 1 default

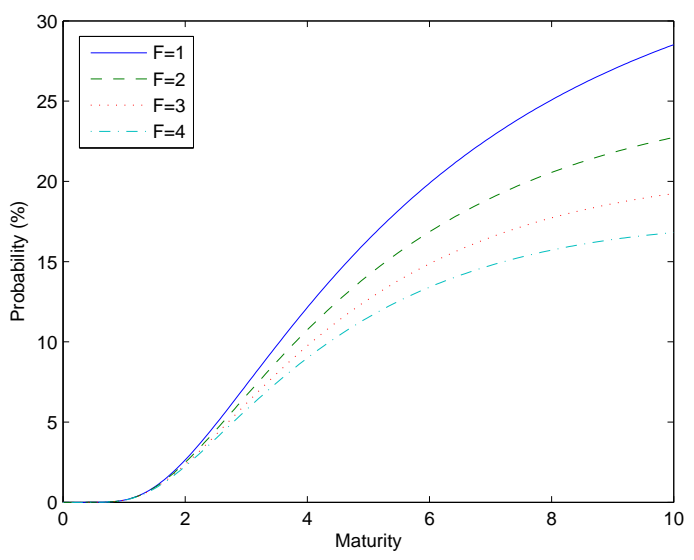

Figure 4: Probability of 2 defaults

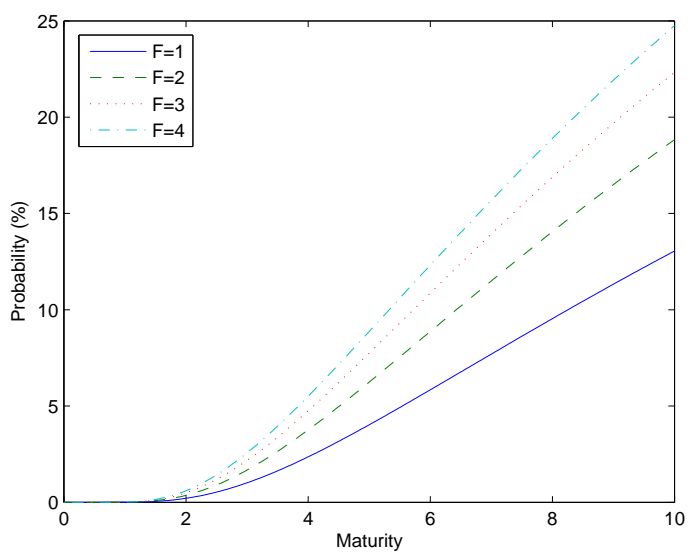

Default probabilities for $\rho=+0.5$

$\sigma_{i}=0.2, r_{f}=0.05, q_{i}=0, \gamma_{i}=0.03$, initial credit quality $=2$

is slightly lower for lower values of $\mathrm{F}$. When the companies are positively correlated, contagion has a greater impact, and since a second default is more likely for higher $\mathrm{F}$, the probability of one default is lower for higher F, while the probability of two defaults is higher.

Since zero correlation corresponds to no contagion, all curves cross at this point. The probability of a second default being higher for positive correlation and contagion, and lower for negative correlation and contagion, explains the position of the curves relative to one another. In Figure 7, for example, for negative correlation, a greater degree of contagion means a second default is less likely, so the probability of exactly one default is greater for higher values of F. Contrastingly, a second default is more likely with higher $\mathrm{F}$ for positive values of correlation and so the probability of one default decreases with increasing $\mathrm{F}$ for positive correlation. The same argument explains the results for the probability of two defaults.

Figure 5: Probability of 1 default

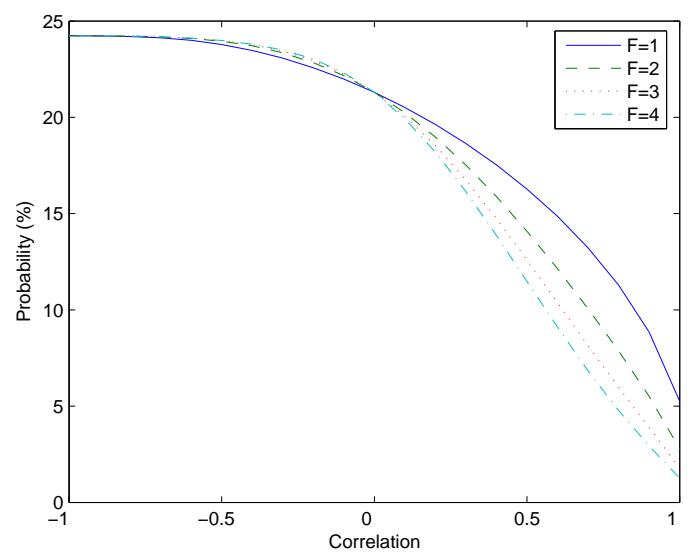

Figure 6: Probability of 2 defaults

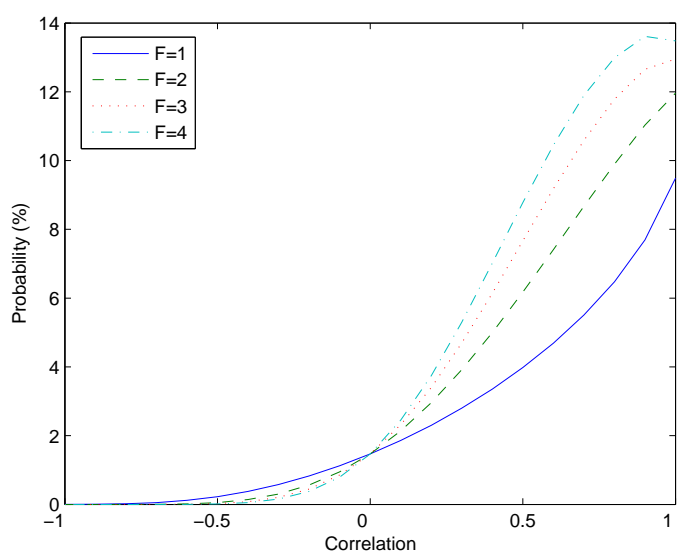

Five-year default probabilities

$\sigma_{i}=0.2, r_{f}=0.05, q_{i}=0, \gamma_{i}=0.03$, initial credit quality $=2$ 
Figure 7: Probability of 1 default

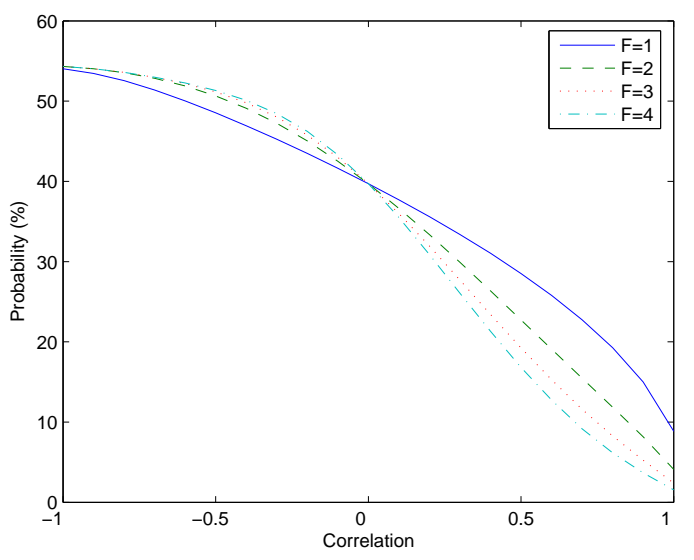

Figure 8: Probability of 2 defaults

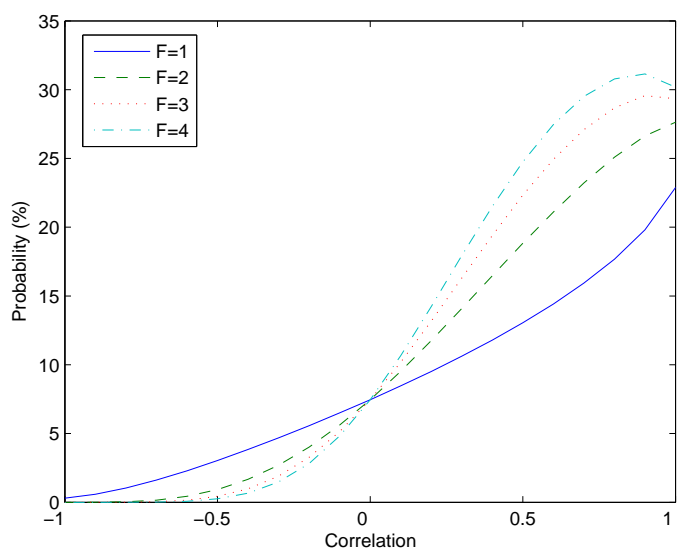

Ten-year default probabilities

$\sigma_{i}=0.2, r_{f}=0.05, q_{i}=0, \gamma_{i}=0.03$, initial credit quality $=2$

In Figures 6 and 8 we see that the probability of two defaults peaks for a value of $\rho<1$ for higher values of $\mathrm{F}$. The same effect is evident and better highlighted in Figures 9 and 10 which illustrate the impact of correlation and contagion on the expected number of defaults over five and ten years.

Figure 9: 5 years

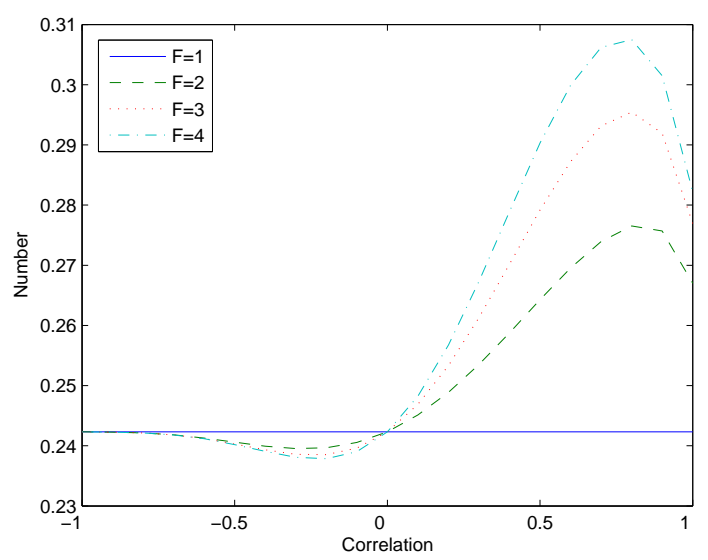

Figure 10: 10 years

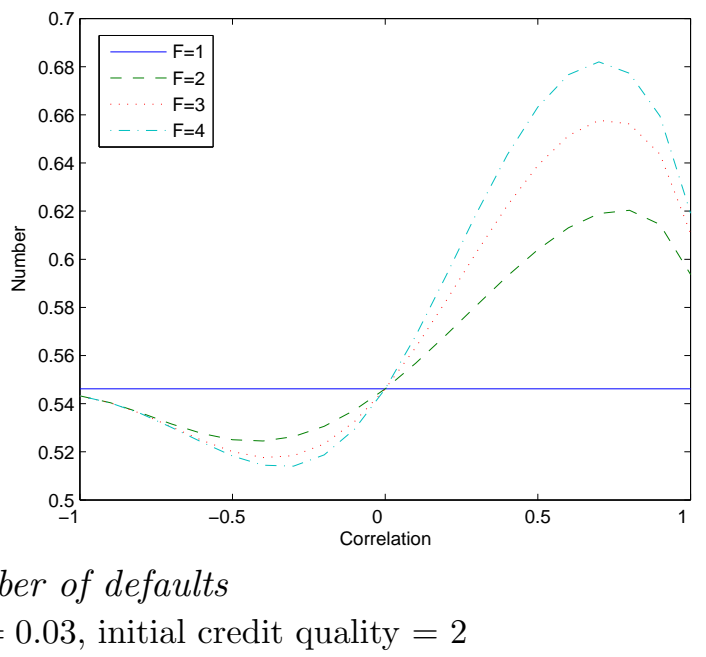

For a given value of $\rho$, the higher $\mathrm{F}$ is, the greater the impact the contagion has on the expected number of defaults, as expected. However, the greatest impact occurs for values of $\rho=\rho_{ \pm}$where $-1<\rho_{-}<0$ and $0<\rho_{+}<1$, and not for $\rho= \pm 1$. As $\rho$ increases, there are conflicting influences on default probabilities. With increasing correlation, the probability of a default decreases, however, if one occurs, the presence of contagion then means that a second default is much more likely for high positive values of $\rho$ and unlikely for negative values of $\rho$. For $\rho_{-}<\rho<\rho_{+}$, the presence of contagion is the driving factor with spreads increasing with correlation. For correlation nearer \pm 1 , the probability of there being a default in the 
first place for contagion to have an effect is more important. In other words, contagion has the biggest impact for $\rho \in\left(\rho_{-}, \rho_{+}\right)$, the most likely levels to occur in reality.

As illustrated in Figures 9 and 10, in the absence of contagion $(\mathrm{F}=1)$, the expected number of defaults is independent of the level of correlation. This follows immediately from the linearity of the expectation operator, and denoting the default indicator process for firm $i$ by $N_{i}$, can be calculated directly from

$$
\mathbb{E}(\text { Number of defaults })=\sum_{i=1}^{2} \mathbb{P}\left(N_{i}=1\right) .
$$

Writing

$$
\begin{aligned}
Y_{i}(t) & =\ln \left(\frac{V_{i}(t)}{V_{i}(0)} e^{-\gamma_{i} t}\right)=\alpha_{i} t+\sigma_{i} W_{i}(t) \\
\alpha_{i} & =r_{f}-q_{i}-\gamma_{i}-\frac{1}{2} \sigma_{i}^{2} \\
B_{i} & =\ln \left(\frac{b_{i}(0)}{V_{i}(0)}\right)
\end{aligned}
$$

then

$$
\begin{aligned}
\mathbb{P}\left(N_{i}=1\right) & =1-\mathbb{P}\left(\underline{Y}_{i}(t) \geq B_{i}\right) \\
& =\Phi\left(\frac{B_{i}-\alpha_{i} t}{\sigma_{i} \sqrt{t}}\right)+e^{2 \alpha_{i} B_{i} / \sigma_{i}^{2}} \Phi\left(\frac{B_{i}+\alpha_{i} t}{\sigma_{i} \sqrt{t}}\right),
\end{aligned}
$$

providing an easy way to check the accuracy of our numerical results ${ }^{6}$.

Figures 11 and 12 show the impact of contagion on five and ten-year second-to-default CDS spreads for different values of correlation. Again, we see the same form, with spreads higher in the presence of contagion for positive correlation and lower for negative correlation, with a peak for some $0<\rho<1$ for positive values of contagion. The more likely a second default is to occur, the riskier the default swap and the greater the spread. Figures 13 and 14 show the same results against maturity for $\rho= \pm 1 / 2$. It is clear from both sets of graphs that the existence and nature of both firm-value correlation and default contagion can have a large impact on CDS spreads.

Our framework for incorporating contagion is flexible enough to enable us to assume that only default by certain companies leads to contagion. This we illustrate in Figures 15 and 16 for the case of two correlated companies, only one of which directly influences the other. For $\mathrm{F}=2$ and $\mathrm{F}=4$, we compare the case in which bankruptcy of either company causes a jump in volatility at the remaining company (labeled 'Double' in the graphs) to the case in which only one company impacts the other (labeled 'Single' in the graphs). In the latter case, if company one defaults, the volatility of company two jumps, but if company two defaults, company one carries on as usual, its volatility unaffected by the default.

Results are as we would expect with asymmetric contagion having less impact on spreads. Considering the relative impact of $\mathrm{F}=2$ Double (symmetric contagion) and $\mathrm{F}=4$ Single (asymmetric contagion) also highlights the non-linear relationship between changes in volatility and

\footnotetext{
${ }^{6}$ Results in Figures 9 and 10 are accurate to five decimal places using ten refinements and 200 time-steps.
} 
Figure 11: 5-year 2nd-to-default CDS

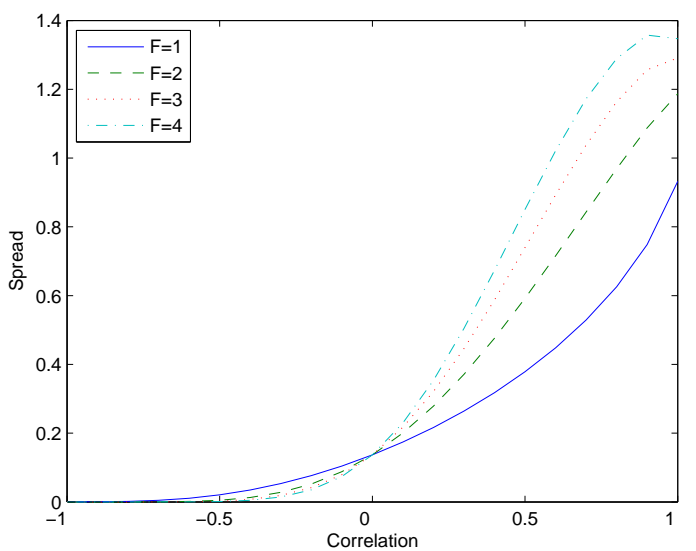

Figure 12: 10-year 2nd-to-default CDS

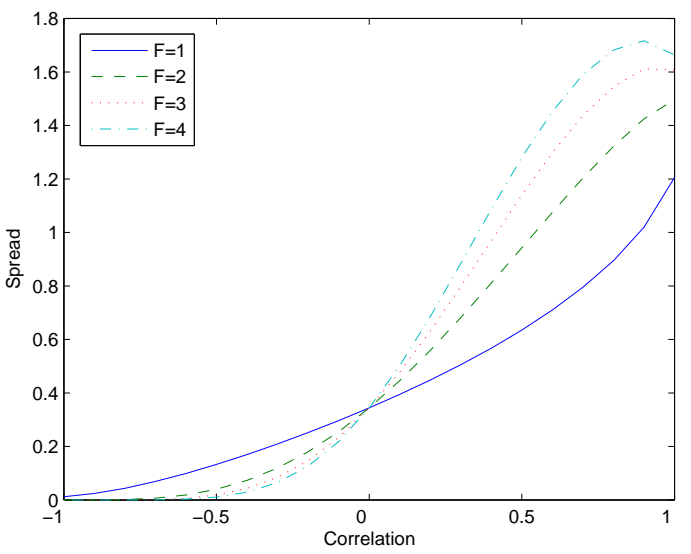

$\sigma_{i}=0.2, r_{f}=0.05, q_{i}=0, \gamma_{i}=0.03$, initial credit quality $=2, \mathrm{R}=0.5$

Figure 13: $\rho=-0.5$

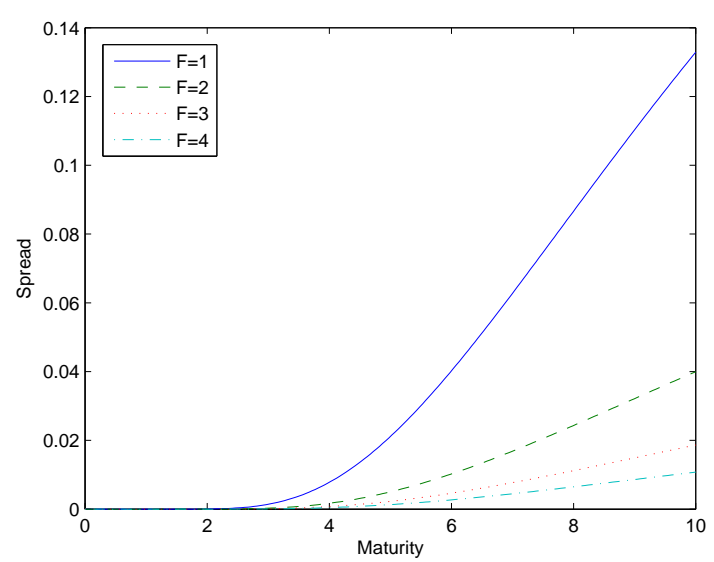

Figure 14: $\rho=+0.5$

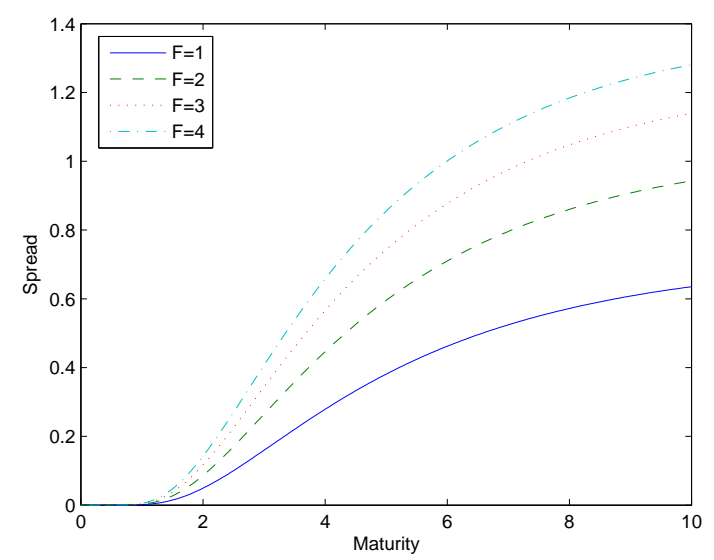

Second-to-default spreads with credit contagion $\sigma_{i}=0.2, r_{f}=0.05, q_{i}=0, \gamma_{i}=0.03$, initial credit quality $=2, \mathrm{R}=0.5$ 
Figure 15: $\rho=-0.5$

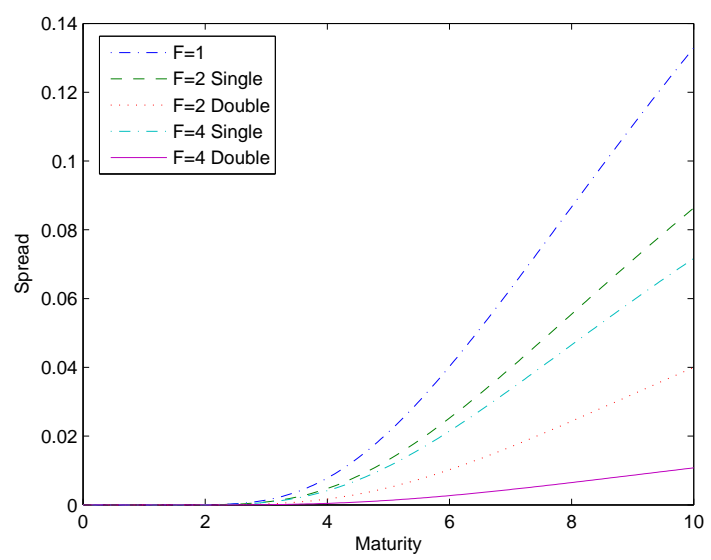

Figure 16: $\rho=+0.5$

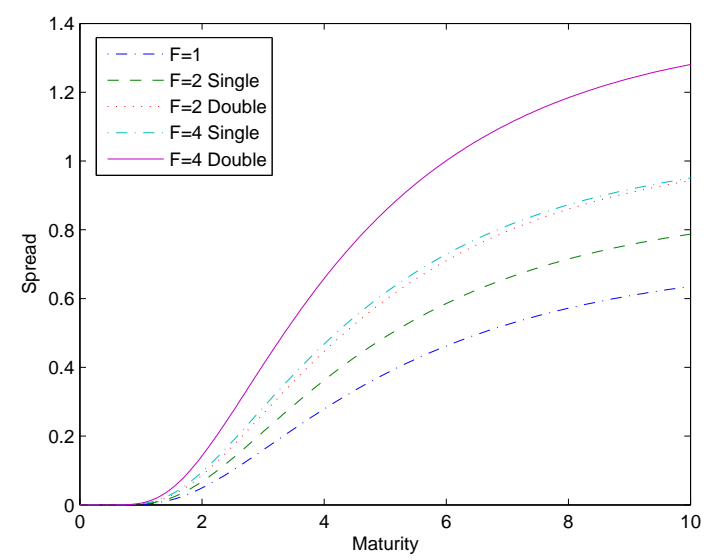

Second-to-default spreads with asymmetric credit contagion

$\sigma_{i}=0.2, r_{f}=0.05, q_{i}=0, \gamma_{i}=0.03$, initial credit quality $=2, \mathrm{R}=0.5$

changes in spreads. For $\rho=-0.5$, symmetric contagion with $\mathrm{F}=2$ has more spread impact than asymmetric contagion with $\mathrm{F}=4$. The reverse is true for $\rho=+0.5$.

Calculating the probability that a given company defaults is also straightforward in this framework and so we can consider the impact of correlated firm values and contagion on the level of default contagion using

$$
\operatorname{Corr}\left[N_{1}(t), N_{2}(t)\right]=\frac{\mathbb{E}\left[N_{1}(t) \cdot N_{2}(t)\right]-\mathbb{E}\left[N_{1}(t)\right] \cdot \mathbb{E}\left[N_{2}(t)\right]}{\sqrt{\operatorname{Var}\left[N_{1}(t)\right] \cdot \operatorname{Var}\left[N_{2}(t)\right]}} .
$$

with $N_{i}$ the default indicator process for firm $i$ as before, and

$$
\begin{aligned}
\mathbb{E}\left[N_{i}(t)\right] & =\mathbb{P}\left(N_{i}(t)=1\right) \\
\operatorname{Var}\left[N_{i}(t)\right] & =\mathbb{P}\left(N_{i}(t)=1\right) \cdot\left[1-\mathbb{P}\left(N_{i}(t)=1\right)\right] \\
\mathbb{E}\left[N_{1}(t) \cdot N_{2}(t)\right] & =\mathbb{E}\left[N_{1}(t)\right]+\mathbb{E}\left[N_{2}(t)\right]-\mathbb{P}\left(N_{1}(t)=1 \text { or } N_{2}(t)=1\right) .
\end{aligned}
$$

Zhou (2001) examines the impact of firm value correlation and firm credit quality on default correlation in the non-contagion setting. Figures 17 and 18 illustrate the impact of contagion for five and ten-year horizons. As expected, the presence of credit contagion increases the range of possible default correlations. When asset correlation is positive, contagion increases default correlation, and when it is negative, contagion causes default correlation to become more negative.

In Figures 19 and 20 we illustrate the term structure of default correlation, with and without contagion. Results can be compared with Figure 1 in Zhou (2001). Using identical parameters to Zhou, we are able to exactly replicate his results using our method. Of note, for our parameter values, over the ten-year time horizon shown, we see no peak in the magnitude of default correlation. This is as a result of the lower value we assign to firm volatility 0.2 compared to 0.4 in Zhou's work. As he explains, the magnitude of default correlation declines over the longer-term since beyond a certain point, the event of non-default becomes idiosyncratic. The effect of reducing volatility or increasing initial credit quality is to move the peak in default correlation further out - beyond ten years in our case. 
Figure 17: 5-year default correlation

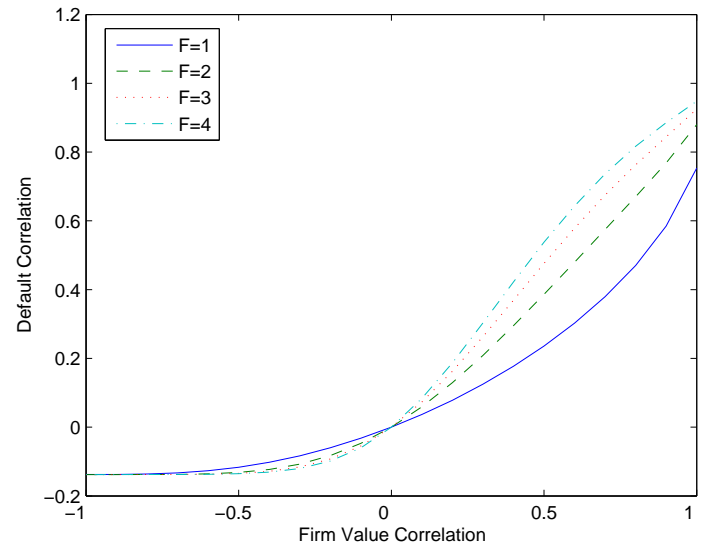

Figure 18: 10-year default correlation

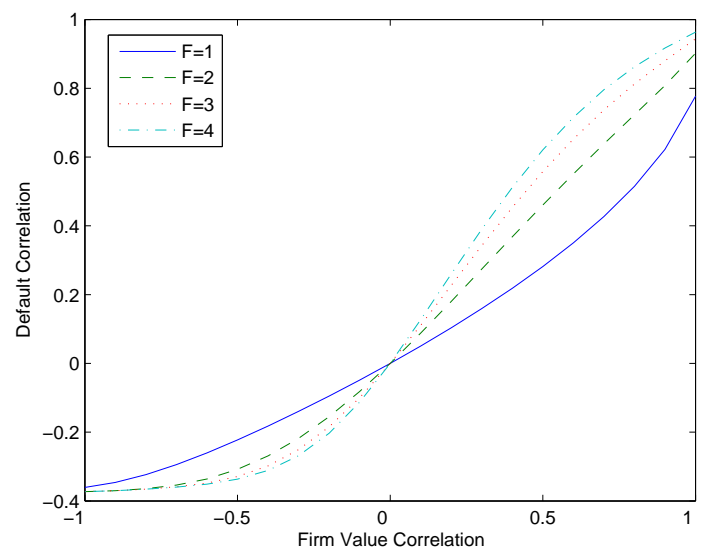

$\sigma_{i}=0.2, r_{f}=0.05, q_{i}=0, \gamma_{i}=0.03$, initial credit quality $=2$
Figure 19: No contagion, $\mathrm{F}=1$

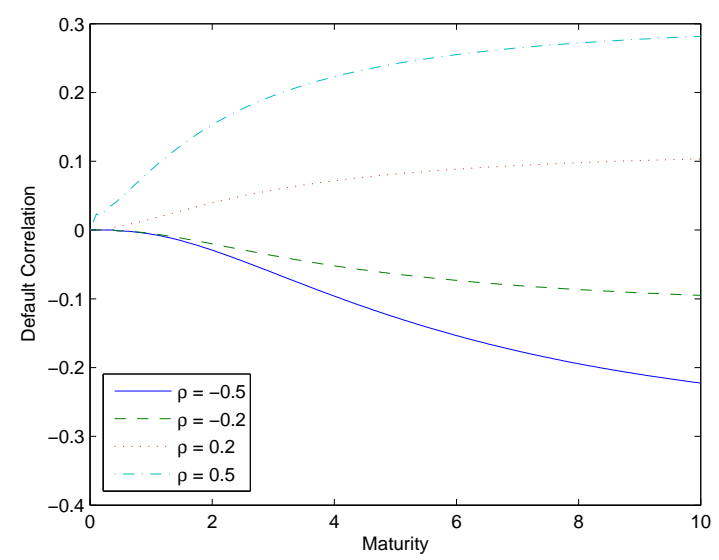

Figure 20: Contagion, $\mathrm{F}=4$

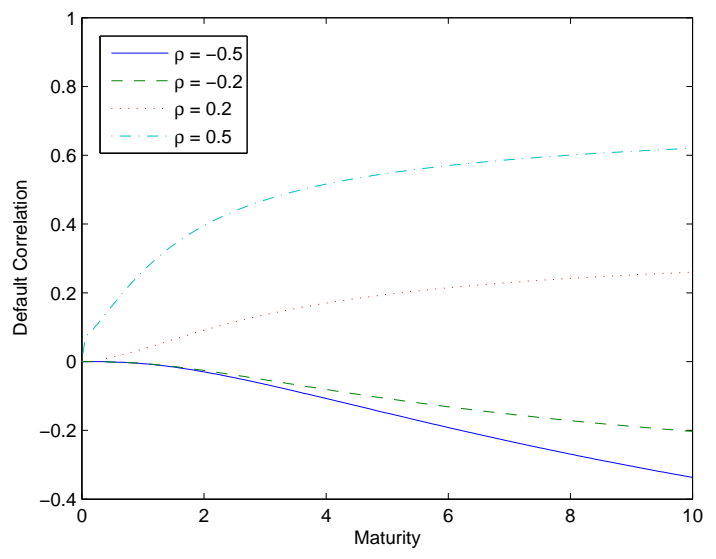

Default correlation with and without contagion $\sigma_{i}=0.2, r_{f}=0.05, q_{i}=0, \gamma_{i}=0.03$, initial credit quality $=2$ 


\section{Contagion with Decay}

Currently, with contagion as specified by (7), the knock-on effect of a default has a lasting impact on remaining companies. Once a firm's volatility jumps to a new level, it stays there forever. This is not necessarily particularly realistic unless the default event serves to permanently change the corporate environment. Rather, it is more usual for contagion to take the form of a decaying spike in spreads or volatility. In the absence of a second default or more news, spreads tend to trend back towards their original level over time.

In the event of default by company $i$, we can incorporate this behavior into our model by having company $j$ 's volatility jump according to

$$
\sigma_{j} \rightarrow \sigma_{j}\left(1+\Delta_{i j} e^{-\zeta\left(t-\tau_{i}\right)}\right) .
$$

In this way, volatility jumps by an amount $\Delta_{i j}$, which can depend on the identity of the defaulting company, and then trends exponentially back to its original level, at a rate determined by the parameter $\zeta$. $\tau_{i}$ denotes the default time of firm $i$ and $j \neq i$. For baskets of $n>2$ companies, the impact of a second or subsequent default would be incorporated similarly. For example, for $k \notin\{i, j\}$, if company $i$ defaulted at time $\tau_{i}$ and then company $k$ defaulted at time $\tau_{k}>\tau_{i}$, the impact on the volatility of firm $j$ would be

$$
\begin{aligned}
\sigma_{j} & \rightarrow \sigma_{j}\left(1+\Delta_{i j} e^{-\zeta\left(t-\tau_{i}\right)}\right) \quad \text { for } \quad \tau_{i} \leq t \leq \tau_{k} \\
& \rightarrow \sigma_{j}\left(1+\Delta_{i j} e^{-\zeta\left(t-\tau_{i}\right)}\right)\left(1+\Delta_{k j} e^{-\zeta\left(t-\tau_{k}\right)}\right) \quad \text { for } \quad t \geq \tau_{k} .
\end{aligned}
$$

Since the extent and direction of the jump in volatility is likely to depend on the identity of the firms in question, it makes sense for $\Delta_{i j}$ to vary, and also for it to be possible for $\Delta_{i j} \neq \Delta_{j i}$ so that there is an asymmetry in the default impact of companies on one another. Similarly, $\zeta$ could be allowed to depend on the firm identities.

Davis and Lo (2001) allow for a similar effect in their model of infectious defaults. They consider large homogeneous portfolios with two risk states - normal and enhanced. Default by one of the companies causes the hazard rate of all remaining companies to jump to an elevated level where it remains for an exponentially distributed length of time before returning to its original level. In their framework there are therefore two jumps in the hazard rate, marking the beginning and the end of the enhanced-risk state. Here, we assume an initial jump in volatility on default, the effect of which then dissipates over time.

Assuming that company $i$ is the first company to default, the volatilities of the remaining firms as given by (11) are then time-dependent, $\sigma_{j}(t), j \neq i$ for $t \geq \tau_{i}$. As outlined in Wilmott (1998), we can remove the time-dependence by replacing $\sigma_{j}^{2}(t)$ with its average over the remaining time-to-maturity, $\bar{\sigma}_{j}^{2}$, where

$$
\begin{aligned}
\bar{\sigma}_{j}^{2} & =\frac{1}{T-\tau_{i}} \int_{0}^{T-\tau_{i}} \sigma_{j}^{2}(s) \mathrm{d} s \\
& =\frac{\sigma_{j}^{2}}{T-\tau_{i}} \int_{0}^{T-\tau_{i}}\left(1+2 \Delta_{i j} e^{-\zeta s}+\Delta_{i j}^{2} e^{-2 \zeta s}\right) \mathrm{d} s \\
& =\sigma_{j}^{2}+\frac{2 \Delta_{i j} \sigma_{j}^{2}}{\zeta\left(T-\tau_{i}\right)}\left(1-e^{-\zeta\left(T-\tau_{i}\right)}\right)+\frac{\sigma_{j}^{2} \Delta_{i j}^{2}}{2 \zeta\left(T-\tau_{i}\right)}\left(1-e^{-2 \zeta\left(T-\tau_{i}\right)}\right) .
\end{aligned}
$$


In the case of a two-firm basket, we can then solve the PDE (2), by breaking the time period into two since the equation decouples on the boundary. We consider the usual two-firm problem on $\left[0, \tau_{i}\right]$ and a one-company problem on $\left[\tau_{i}, T\right]$. By standard first passage theory, as given in Musiela and Rutkowski (1998), the probability that company $j$ survives and does not fall below its default threshold before maturity $T$ given that company $i$ defaults at time $\tau_{i}<T$ is

$$
\Phi\left(\frac{X_{j}\left(\tau_{i}\right)+\bar{\alpha}_{j}\left(T-\tau_{i}\right)}{\bar{\sigma}_{j} \sqrt{T-\tau_{i}}}\right)-e^{\left\{-2 \bar{\alpha}_{j} X_{j}\left(\tau_{i}\right) / \bar{\sigma}_{j}^{2}\right\}} \Phi\left(\frac{-X_{j}\left(\tau_{i}\right)+\bar{\alpha}_{j}\left(T-\tau_{i}\right)}{\bar{\sigma}_{j} \sqrt{T-\tau_{i}}}\right),
$$

where $\bar{\alpha}_{j}=r_{f}-q_{j}-\gamma_{j}-\bar{\sigma}_{j}^{2} / 2$ and $X_{j}(t)=\ln \left(V_{j}(t) e^{-\gamma_{j} t} / b_{j}(0)\right)$.

We then solve $(2)$ on $\left[0, \tau_{i}\right]$ exactly as before, subject to additional boundary conditions at $X_{i}(t)=0$ given by (12). By modifying the initial condition and specifying the boundary conditions according to whether we are interested in company $j$ surviving or defaulting, we are able to calculate a range of default probabilities as before, and asymmetric default contagion can be easily incorporated.

For consistency with the framework in Section 3, we set $\Delta=F^{\rho}-1$, so that the initial jump in volatility is driven by both the degree of contagion, $F$, and the correlation between firm values, as before ${ }^{7}$. By specifying the process in this manner we can directly compare results with and without decay as shown in Figures $21-28$.

In Figures 21 - 24, we consider results for no contagion $(\mathrm{F}=1, \zeta=0)$, contagion but no decay $(\mathrm{F}=4, \zeta=0)$ and contagion with varying rates of decay $(\mathrm{F}=4, \zeta=0.5,2,4, \& 8)$. Values of $\zeta$ of 8,4 and 2 correspond to volatility reverting to its pre-default level over roughly six months, one year and two years, respectively. For the purpose of comparison, we also include the fairly extreme case of $\zeta=0.5$ for which the effects of the default take nearly ten years to dissipate. In practice for a company's default to have such a lasting impact, it would have to have been a key company in the sector or market of interest, or a particularly momentous default. The possible bankruptcy of General Motors and the fall-out following the Enron debacle spring to mind as incidences that might lead to such a long-drawn out settling down of spreads.

Figures 21 and 22 show the probability of one and two defaults, respectively, for a ten-year period. We see that results with a decay in default contagion, $\zeta>0$, have the same form and lie closer to results with no contagion (shown by the solid black line) than those with a high degree of permanent contagion, $\zeta=0$. As $\zeta$ increases, the rate at which the contagion effects dissipate increases and probabilities tend more quickly towards their non-contagion level.

Since the rate of decay is exponential, it makes sense that the impact of the contagion is fairly limited when compared to the situation in which the jump in volatility is permanent. That said, as we see from the 2nd-to-default spreads in Figures 23 and 24, the effect is still large enough to warrant attention. For highly correlated companies, even if the knock-on impact of the default dissipates in six months $(\zeta=8)$, spreads are five to ten basis points higher with contagion. The spread impact of contagion for negative values of $\rho$ is considerably lower than for positive $\rho$. This is because for $\rho<0$ contagion serves to reduce volatility and increasing volatility by a given amount (e.g. from 0.2 to 0.3 ) has a much bigger impact on spreads than reducing volatility by the same amount (0.2 to 0.1$)$.

\footnotetext{
${ }^{7}$ As in Section 3, we denote $\rho_{12}=\rho$ and so $\Delta$ is the same for both companies.
} 
Figure 21: Probability of 1 default

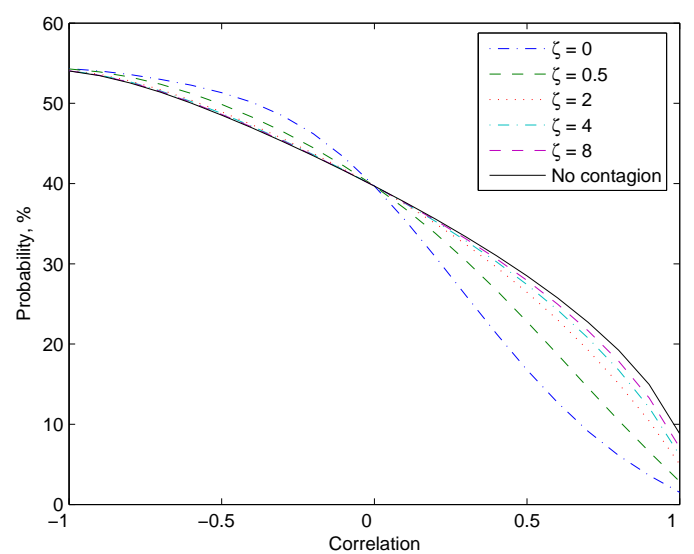

Figure 22: Probability of 2 defaults

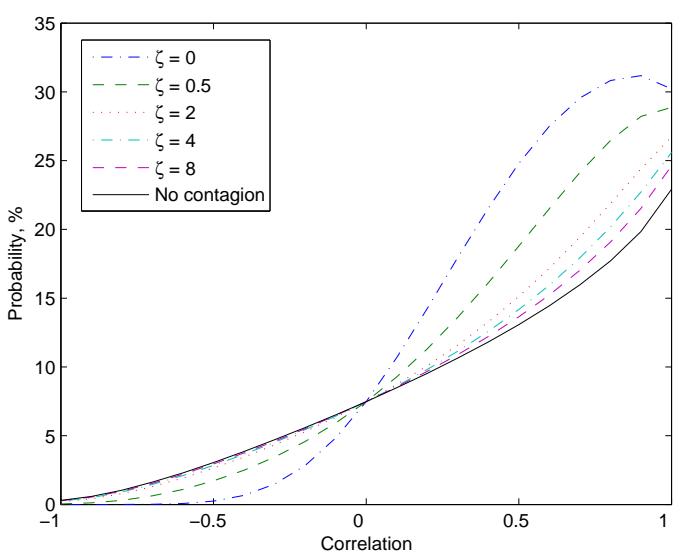

Ten-year default probabilities with decaying contagion $\sigma_{i}=0.2, r_{f}=0.05, q_{i}=0, \gamma_{i}=0.03$, initial credit quality $=2$

Figure 23: 10-year 2nd-to-default CDS

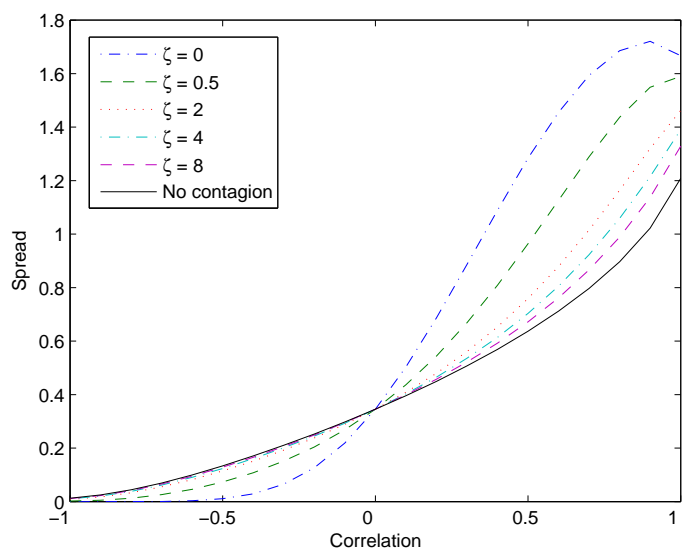

Figure 24: Spread curve for $\rho=0.5$

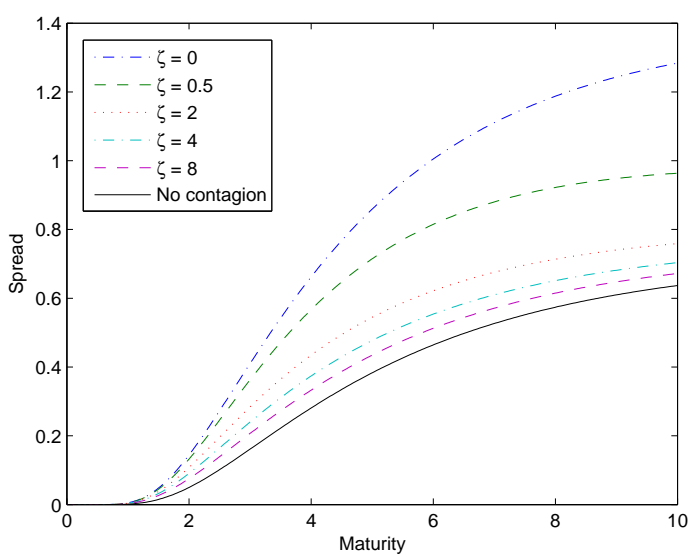

2nd-to-default CDS spreads with decaying contagion $\sigma_{i}=0.2, r_{f}=0.05, q_{i}=0, \gamma_{i}=0.03$, initial credit quality $=2, \mathrm{R}=0.5$ 
Figures $25-28$ show the direct impact of contagion on spreads for positive values of correlation. Figures $25-26$ give results for $\rho=0.5$, Figures $27-28$ for $\rho=0.75$. In each case we illustrate the extra spread in basis points (1/100ths of a percent) for 2 nd-to-default CDSs with contagion compared to the base case of correlated firm values but no default contagion. For reference, in the absence of contagion, the five-year spread with $\rho=0.5$ is 38 basis points, whilst for $\rho=0.75$ it is 63 basis points. The extra spreads we are seeing due to the existence of contagion are therefore of a meaningful size.

Figure 25:

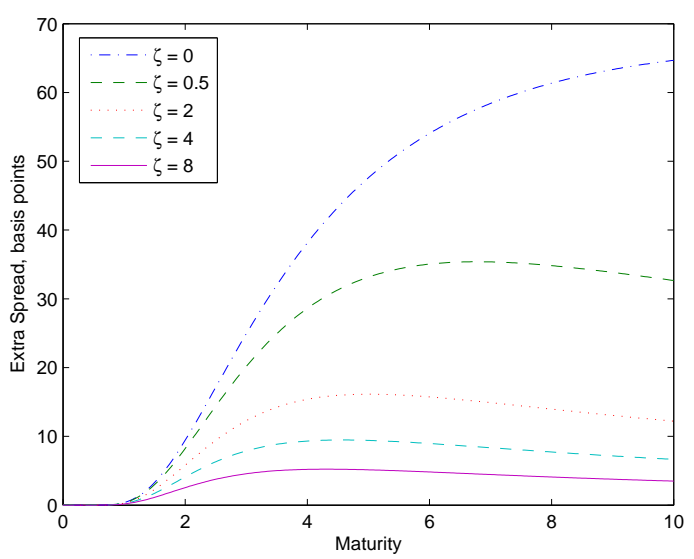

Figure 26:

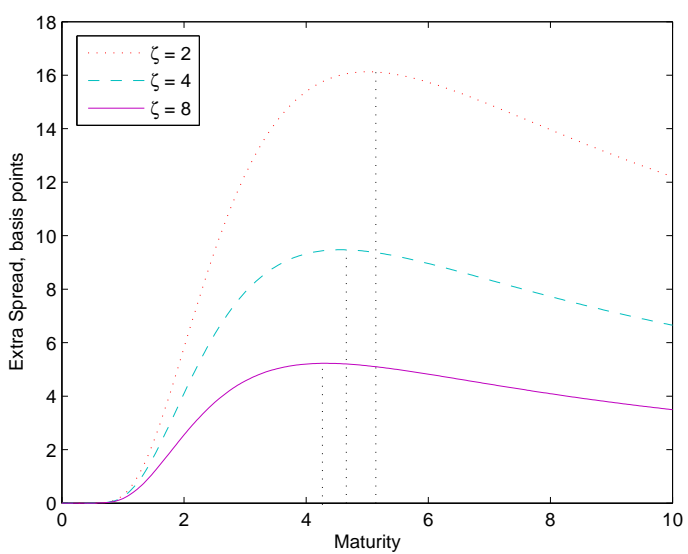

Additional spread on 2nd-to-default CDS due to contagion, $\rho=0.5$ $\sigma_{i}=0.2, r_{f}=0.05, q_{i}=0, \gamma_{i}=0.03$, initial credit quality $=2, \mathrm{R}=0.5$

Figure 27:

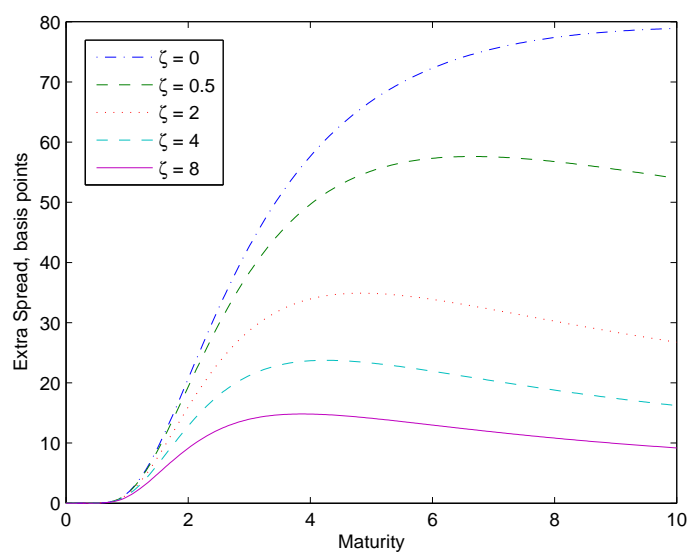

Figure 28:

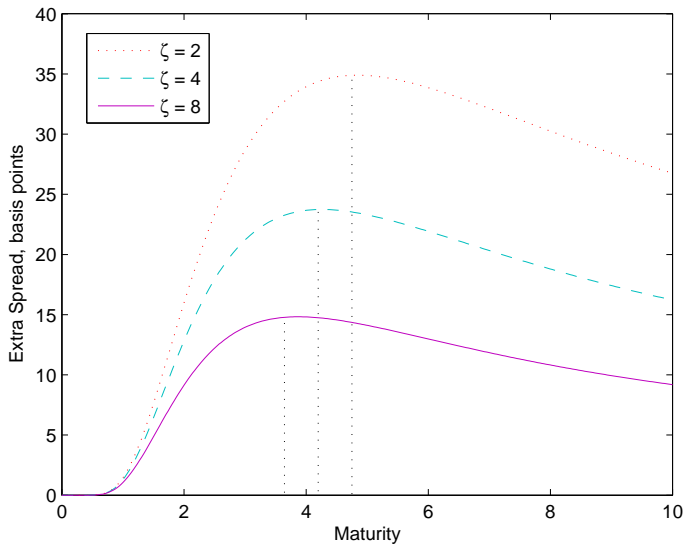

Additional spread on 2nd-to-default CDS due to contagion, $\rho=0.75$ $\sigma_{i}=0.2, r_{f}=0.05, q_{i}=0, \gamma_{i}=0.03$, initial credit quality $=2, \mathrm{R}=0.5$

From the left hand graphs, we see that when contagion results in a permanent increase in volatilities $(\zeta=0)$, spreads increase with maturity - the longer dated the CDS, the greater the impact of contagion. This is not the case when the jump in volatility declines over time. As would be expected, the faster the rate of decay, the shorter the maturity of the product that sees the greatest spread impact, and the earlier we see a peak in the spread difference. 
This is easier to see in the right hand graphs, Figures 26 and 28. These reproduce the lower three curves of the left hand graphs and show clearly the peaks for $\zeta=2,4$ and 8 . For $\rho=0.75$, contagion with $\zeta=2$ has the greatest impact on spreads for a 4.9-year maturity CDS whilst when $\zeta=8$, the difference is greatest for a maturity of 3.9 years.

\section{$5 \quad$ Results for Three Firms}

Using identical numerical techniques as in Section 3, we can generate $k^{\text {th }}$-to-default spreads for baskets of three companies. We begin, as in the two-firm case, by showing results for the joint survival probability and first-to-default CDS spread in Figures 29 and 30. Comparing with Figures 1 and 2 for the two-company basket, we see the same shaped curves. As would be expected, survival probabilities are lower and spreads are higher when there are three firms as the presence of the extra company increases the risk of the product.

Figure 29: Joint survival probability

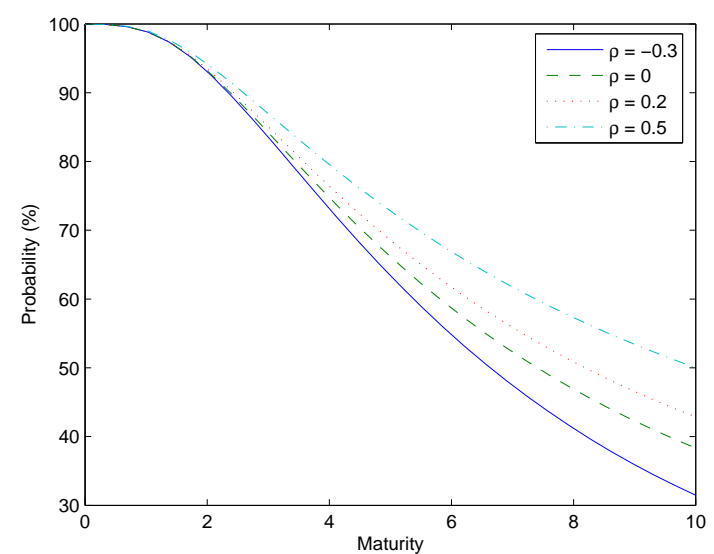

Figure 30: First-to-default CDS spread

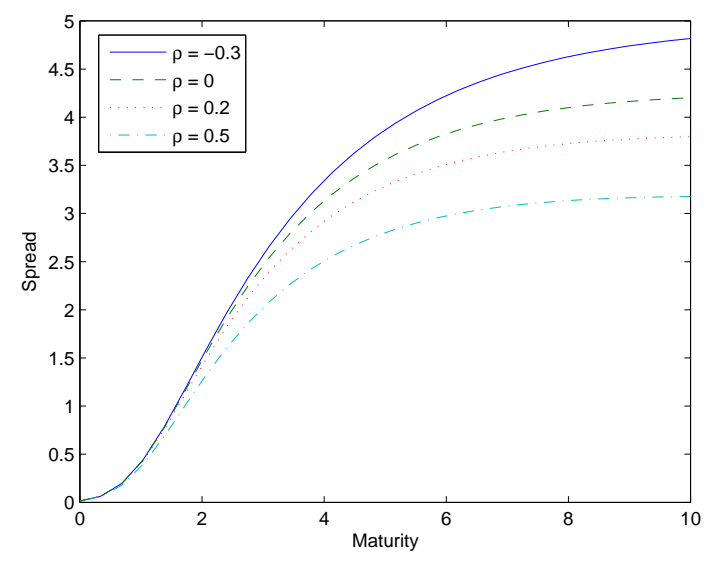

$\sigma_{i}=0.2, r_{f}=0.05, q_{i}=0, \gamma_{i}=0.03$, initial credit quality $=2, \mathrm{R}=0.5$

In Figures 29 - 34, we are assuming that all three firms have identical characteristics, given by the parameters shown, and that there is just one correlation variable, $\rho_{i j}=\rho, i \neq j$. Considering homogeneous firms in the first instance enables us to see the direct spread impact of changes in correlation and default contagion.

Figures 31 and 32 show second-to-default spreads for various values of correlation, with and without contagion. As before, spreads are the same in each case for zero correlation, lower with contagion for negatively correlated firms and higher with contagion when firms are positively correlated. The same results are illustrated in Figures $33-34$ for third-to-default spreads. Spreads are greatest for first-to-default products and lowest for third-to-default products, as they should be.

Finally, in Figures 35 and 36, we provide some results for different correlation structures. In order of increasing spreads, Figure 35 compares first-to-default swap spreads for a twofirm basket with correlation of $0.5(2 \mathrm{D}: \rho=0.5)$, a three-firm basket with correlation of 0.5 between all firms (3D: $\rho=0.5)$, a three-firm basket with two correlated and one completely uncorrelated firm $\left(3 \mathrm{D}: \rho_{12}=0.5, \rho_{23}=\rho_{13}=0\right)$ and a three-firm basket with positively and 
Figure 31: No contagion, $\mathrm{F}=1$

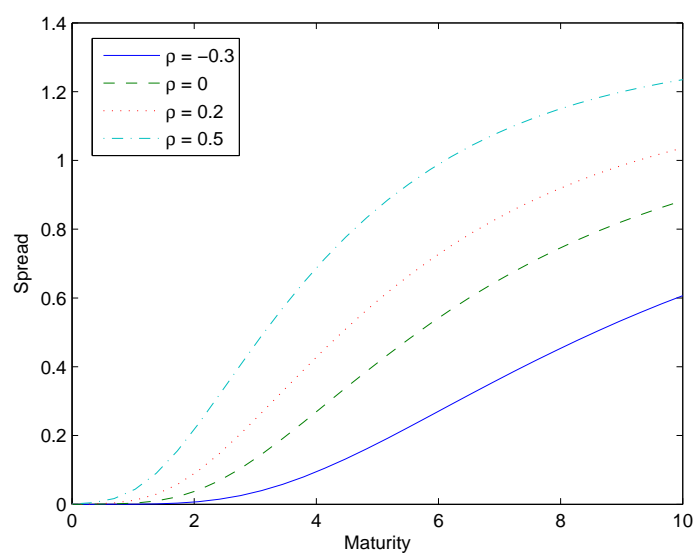

Figure 32: Contagion, $\mathrm{F}=4$

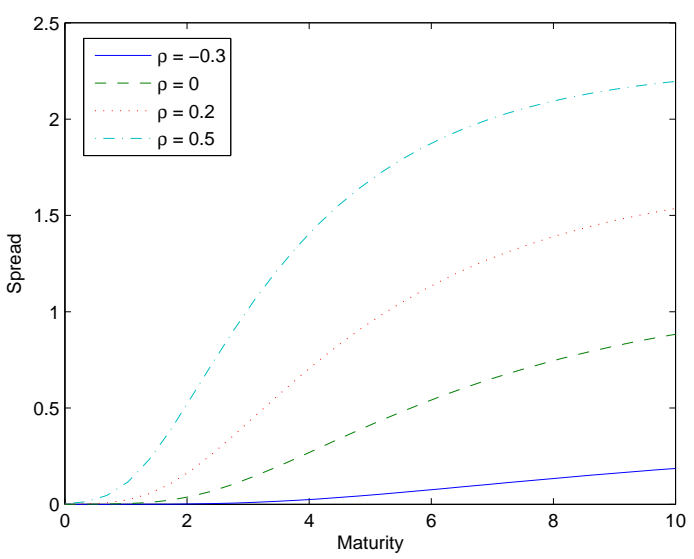

2nd-to-default CDS spreads

$\sigma_{i}=0.2, r_{f}=0.05, q_{i}=0, \gamma_{i}=0.03$, initial credit quality $=2, \mathrm{R}=0.5$

Figure 33: No contagion, $\mathrm{F}=1$

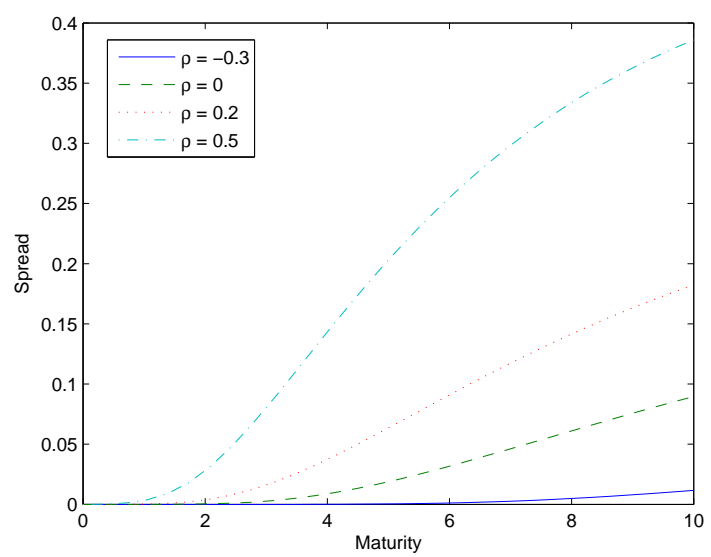

Figure 34: Contagion, $\mathrm{F}=4$

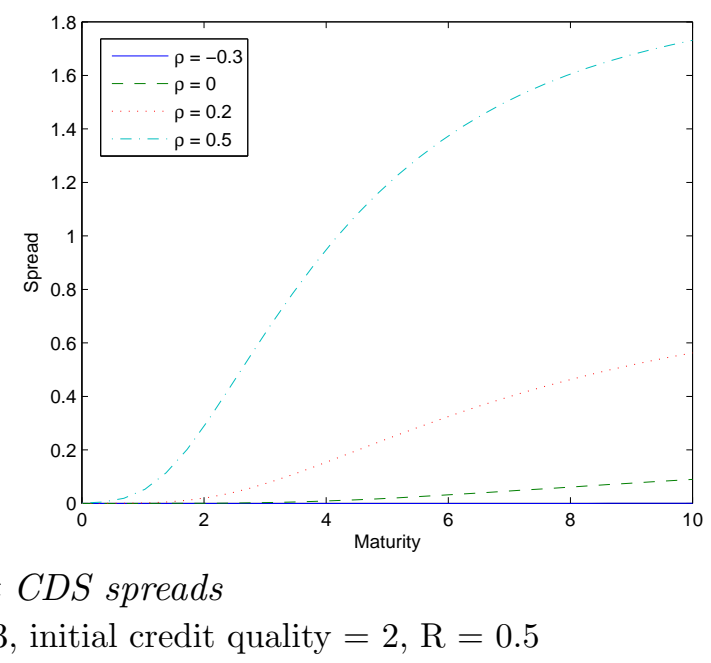


Figure 35: 1st-to-default CDS spread

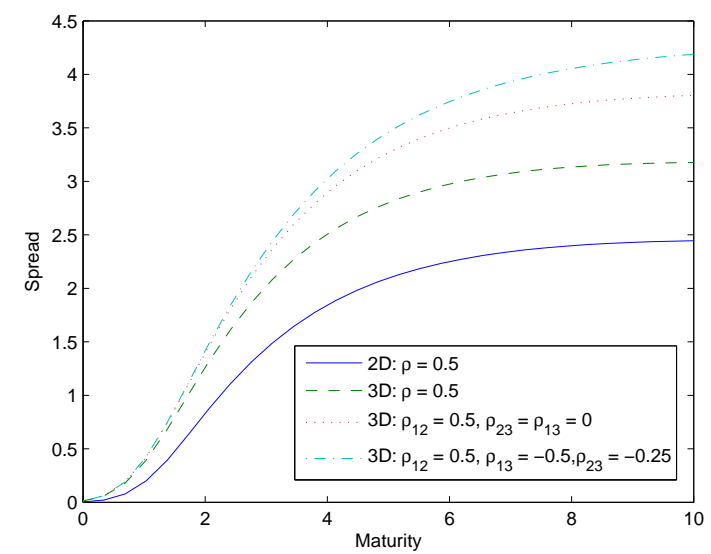

Figure 36: 2nd-to-default CDS spread

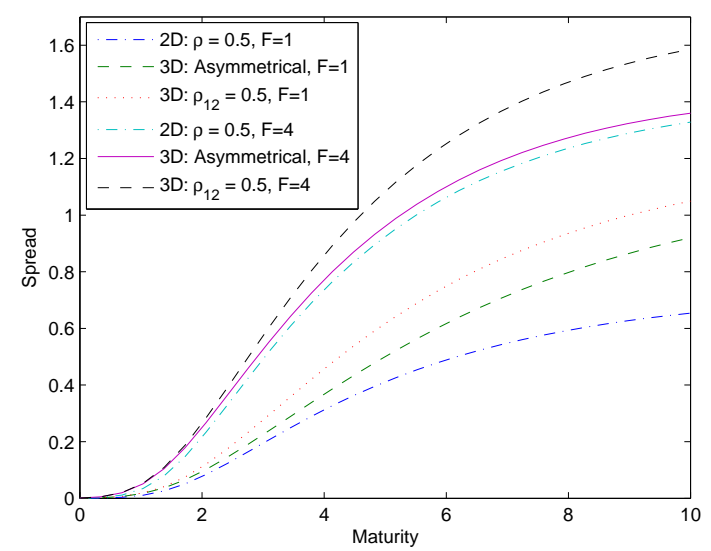

$\sigma_{i}=0.2, r_{f}=0.05, q_{i}=0, \gamma_{i}=0.03$, initial credit quality $=2, \mathrm{R}=0.5$

3D: Asymmetrical corresponds to $\rho_{12}=0.5, \rho_{13}=-0.5, \rho_{23}=-0.25$

$3 \mathrm{D}: \rho_{12}=0.5$ corresponds to $\rho_{12}=0.5, \rho_{23}=\rho_{13}=0$.

negatively correlated firms (3D: $\left.\rho_{12}=0.5, \rho_{13}=-0.5, \rho_{23}=-0.25\right)$. The order is intuitive, but it is interesting to note the relative differences in spreads and the impact that varying the correlation structure can have. Figure 36 gives similar results for second-to-default swap spreads, considering, in addition, the impact of contagion $(\mathrm{F}=4$ versus $\mathrm{F}=1)$. Once again, these graphs highlight the flexibility of the model for investigating the relationship between the dependence structure and spreads.

We are restricted by memory constraints to using a maximum of six refinements in our numerical evaluation of three-dimensional results. This means that calculations are very fast (the number of refinements is the limiting factor in the accuracy of results rather than the number of time-steps) but are not quite as accurate as we would like ${ }^{8}$. Whilst our numerical method is currently not quite accurate enough to be used for pricing three-firm baskets, the model nonetheless represents a powerful tool for considering the sensitivity of CDS spreads to assumptions regarding input parameters and the dependence structure.

Incorporating decaying contagion in three (or more) dimensions is not as straightforward as in the two-dimensional case, but could be done recursively, starting with the case when all bar one of the firms have defaulted to define the boundary conditions for the boundary planes (as done in Section 4 for the two-dimensional case), and then solving the two-firm case on these planes to give the boundary conditions in three-dimensions.

\section{General $m$-Factor Framework}

Numerical limitations aside, the framework has the flexibility to enable a very general specification of firm value dynamics and underlying correlation structure. Supposing that firm

\footnotetext{
${ }^{8}$ By comparing results for $\mathbb{P}\left(\tau_{1}<T\right)$ calculated in the two and three-dimensional case and stressing the survival probabilities and integrals used in (9) accordingly, the maximum absolute error was around 10 basis points, usually much less.
} 
values are driven by both a number of macro factors and an idiosyncratic factor, with correlation between firms arising from their individual exposures to the macro driving factors, then for $n$ companies, values $V_{i}(t)$, with idiosyncratic factors $W_{i}(t)$ and $m$ independent macro driving processes $Y_{j}(t)$,

$$
\begin{aligned}
d V_{i}(t) & =\left(r_{f}-q_{i}\right) V_{i}(t) d t+\delta_{i} V_{i}(t) d W_{i}(t)+\sum_{j=1}^{m} \gamma_{i j} V_{i}(t) d Y_{j}(t) \\
& =\beta_{i} V_{i}(t) d t+\sum_{j=1}^{d} \eta_{i j} V_{i}(t) d Z_{j}(t)
\end{aligned}
$$

where $d=m+n, \beta_{i}=r_{f}-q_{i}$,

$$
\eta_{i j}=\left\{\begin{array}{llll}
\delta_{i} & \& & d Z_{j}(t)=d W_{j}(t) & \text { for } \quad 1 \leq j \leq n, \quad i=j \\
0 & \& & d Z_{j}(t)=d W_{j}(t) & \text { for } \quad 1 \leq j \leq n, \quad i \neq j \\
\gamma_{i(j-n)} & \& & d Z_{j}(t)=d Y_{(j-n)}(t) & \text { for } \quad n+1 \leq j \leq d,
\end{array}\right.
$$

and for each $i$ and all $j$, the Brownian motions $W_{i}(t)$ and $Y_{j}(t)$ are uncorrelated with one another. $\beta_{i}$ represents the expected growth rate of the value of firm $i$ and the weightings $\gamma_{i j}$ and $\delta_{i}$ represent the exposure of company $i$ to the various macro and idiosyncratic factors. The larger a given weighting is, the greater that factor's influence and the potentially more volatile the company. As before, we assume that company $i$ defaults the first time that its value drops below the level of its default barrier $b_{i}(t)$. Defined in this way, company values can be considered to be driven by both company-specific and global factors. The latter could, for example, be at the country, sector or industry level, introducing a rich correlation structure between companies.

If $\mathbf{V}$ is the vector of firm values, for a function $U(\mathbf{V}, t)$, the infinitesimal generator of (13) is

$$
\mathcal{L} U=\frac{\partial U}{\partial t}+\sum_{i=1}^{n} \beta_{i} V_{i} \frac{\partial U}{\partial V_{i}}+\frac{1}{2} \sum_{i, j=1}^{n} a_{i j} V_{i} V_{j} \frac{\partial^{2} U}{\partial V_{i} \partial V_{j}}
$$

where

$$
a_{i j} d t=\sum_{k, l=1}^{d} \eta_{i k} \eta_{j l}\left\langle d Z_{k}, d Z_{l}\right\rangle
$$

and $\left\langle d Z_{k}, d Z_{l}\right\rangle$ represents the quadratic covariation between $d Z_{k}$ and $d Z_{l}$.

Applying the Feynman-Kac formula as before, we can then calculate the probability of $k$ defaults in $[0, T]$ by solving

$$
\begin{aligned}
\frac{\partial U}{\partial t} & +\sum_{i=1}^{n} \beta_{i} V_{i} \frac{\partial U}{\partial V_{i}}+\frac{1}{2} \sum_{i, j=1}^{n} a_{i j} V_{i} V_{j} \frac{\partial^{2} U}{\partial V_{i} \partial V_{j}}=0 \\
U(\mathbf{V}, T) & =\mathbb{I}_{\Omega_{k}}(\mathbf{V}),
\end{aligned}
$$

where $\Omega_{k}$ is the set corresponding to exactly $k$ companies defaulting in $[0, T]$.

The basic structure of (13) is related to that considered by Hull et al. (2005) in which the value of company $i$ 's assets evolves according to

$$
d \ln V_{i}=\mu_{i} d t+\alpha_{i}(t) \sigma_{i} d F(t)+\sqrt{1-\alpha_{i}(t)^{2}} \sigma_{i} d U_{i}(t)
$$


for common and idiosyncratic Brownian motions $F(t)$ and $U_{i}(t)$. The correlation structure is driven by the $\alpha_{i}(t) \mathrm{s}$, which may be either stochastic or time-dependent, but there is no mechanism for introducing any type of default contagion or inter-company ties in their model.

\section{Conclusion}

We have proposed a numerical approach to modeling firm value dynamics and the default event, enabling valuation of basket credit default swap spreads in a first passage framework with both asset correlation and default contagion. The approach is easy to implement and enables specification of a rich dependence structure incorporating asymmetries and default causality. By modifying the initial condition, there is great flexibility to calculate many different default and survival probabilities of interest, allowing evaluation of $k^{\text {th }}$-to-default CDS spreads, the expected number of defaults and default correlations.

Results reiterate the need for credit models to take into account a full dependence structure, with default contagion having a very clear impact on spreads. Whilst the accuracy of the numerical approach is not quite good enough for pricing baskets of three or more companies and memory constraints prohibit extension to large baskets using current methodology, the model could be used as a powerful tool for analysing the spread impact of different dependence assumptions and parameter values.

The goal remains to extend the approach to cope with bigger baskets in order to price large $k^{\text {th }}$-to-default CDS products and CDO tranches. We have the framework for specifying the dynamics and a realistic dependence structure, but extending the numerics to deal with higher dimensions has proven elusive using finite difference methods ${ }^{9}$. Current work includes applying Monte Carlo techniques to the framework with the goal to model large portfolios of up to 125 companies by dimension reduction using principal component analysis.

Extending the framework to a non geometric Brownian motion setting would be relatively straightforward. It would be interesting to see the impact of incorporating stochastic correlation or stochastic volatility, although this would raise the added issue of market incompleteness. By relating firm correlations or volatilities to a global state variable, it would be possible to have default correlations depend on the state of the economy as considered by Hull et al. (2005), reflecting the fact that defaults tend to be more highly correlated when default probabilities are higher. Another desirable extension would be to incorporate jumps into the specification of the underlying firm value dynamics to remove the predictable nature of default and the resultant zero short spreads. In all cases, results need to be related to those seen in practice, with an evaluation of the ability of different model specifications to explain realistic spread behavior.

\section{References}

M. Baxter. Dynamic modelling of single-name credits and CDO tranches. Nomura Fixed Income Quant Group, 2006.

\footnotetext{
${ }^{9}$ We tried many techniques to increase the speed and accuracy including sparse grid methods (Reisinger and Wittum (2004)), coordinate transformations and singularity removal methods, but with limited success.
} 
http : //www.nomura.com/resources/europe/pdfs/cdomodelling.pdf.

F. Black and J. Cox. Valuing corporate securities: Some effects of bond indenture provisions. Journal of Finance, 31:351-367, 1976.

U. Cherubini, E. Luciano, and W. Vecchiato. Copula methods in finance. Wiley, 2004.

M. Davis and V. Lo. Modelling default correlation in bond portfolios. Working Paper, Imperial College, London., 2001. http : //www.ma.ic.ac.uk/mdavis/docs/mastering_risk.pdf.

C. Finger, V. Finkelstein, G. Pan, J. Lardy, T. Thomas, and J. Tierney. Creditgrades ${ }^{T M}$. Technical Document, RiskMetrics Group, Inc., 2002.

http : //www.creditgrades.com/resources/pdf/CGtechdoc.pdf.

K. Giesecke. Credit risk modeling and valuation: An introduction. Working Paper, Cornell University, 2004.

http://www.stanford.edu/dept/MSandE/people/faculty/giesecke/introduction.pdf.

H. Haworth, C. Reisinger, and W. Shaw. Modelling bonds and credit default swaps using a structural model with contagion. Working paper, Oxford University, 2006. http : //www.maths.ox.ac.uk/ haworthh/Webfiles/2DArticle.pdf.

J. Hull, M. Predescu, and A. White. The valuation of correlation-dependent credit derivatives using a structural model. Working Paper, University of Toronto, 2005. http://www.rotman.utoronto.ca/`hull/DownloadablePublications/StructuralModel.pdf.

J. Hull and A. White. Valuing credit default swaps II: Modeling default correlations. Working Paper, University of Toronto, 2000. http : //www.stern.nyu.edu/fin/workpapers/papers00/wpa00022.pdf.

R. Jarrow and J. Yu. Counterparty risk and the pricing of defaultable securities. The Journal of Finance, LVI No. 5:1765-1800, 2001.

D. Lando. Credit Risk Modeling: Theory 83 Applications. Princeton University Press, 2004.

E. Luciano and W. Schoutens. A multivariate jump-driven financial asset model. ICER Working Paper, 2005.

http : //perswww.kuleuven.be/ u0009713/multivg.pdf.

R. Merton. On the pricing of corporate debt: The risk structure of interest rates. Journal of Finance, 29:449-470, 1974.

T. Moosbrucker. Pricing CDOs with correlated Variance Gamma distributions. Working Paper, University of Cologne, 2006.

http : //www.fmpm.ch/docs/9th/papers_2006_web/9112b.pdf.

M. Musiela and M. Rutkowski. Martingale Methods in Financial Modelling. Springer, 1998.

C. Reisinger and G. Wittum. On multigrid for anisotropic equations and variational inequalities. Computing and Visualization in Science, 7:189-197, 2004.

P.J. Schönbucher. Credit derivatives pricing models. Wiley, 2003.

P. Wilmott. Derivatives: The theory and practice of financial engineering. Wiley, 1998. 
C. Zhou. An analysis of default correlations and multiple defaults. The Review of Financial Studies, 14:555-576, 2001. 